\title{
The proprotein convertase furin is required for trophoblast syncytialization
}

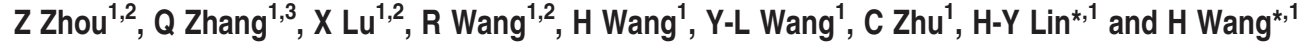

The multinucleated syncytial trophoblast, which forms the outermost layer of the placenta and serves multiple functions, is differentiated from and maintained by cytotrophoblast cell fusion. Deficiencies in syncytial trophoblast differentiation or maintenance likely contribute to intrauterine growth restriction and pre-eclampsia, two common gestational diseases. The cellular and molecular mechanisms governing trophoblast syncytialization are poorly understood. We report here that the proprotein convertase furin is highly expressed in syncytial trophoblast in the first trimester human placentas, and expression of furin in the syncytiotrophoblast is significantly lower in the placentas from pre-eclamptic patients as compared with their gestational age-matched control placentas. Using multiple experimental models including induced fusion of choriocarcinoma BeWo cells and spontaneous fusion of primary cultured cytotrophoblast cells or placental explants, we demonstrate that cytotrophoblast cell fusion and syncytialization are accompanied by furin expression. Furin-specific siRNAs or inhibitors inhibit cell fusion in BeWo cells, as well as trophoblast syncytialization in human placental explants. Furthermore, type 1 IGF receptor (IGF1R) is indicated in this study as a substrate of furin, and processing of IGF1R by furin is an essential mechanism for syncytialization. Finally, using lentivirus-mediated RNAi targeting to mouse trophectoderm, we demonstrate that furin function is required for the development of syncytiotrophoblast structure in the labyrinth layer, as well as for normal embryonic development.

Cell Death and Disease (2013) 4, e593; doi:10.1038/cddis.2013.106; published online 18 April 2013

Subject Category: Experimental Medicine

A hallmark of mammalian reproduction is the fetal-maternal interaction during embryonic development. The placenta, which develops from the extraembryonic trophectoderm (TE), is the essential platform mediating this interaction. After implantation, placental villous cytotrophoblast cells (CTBs), a population of progenitor cells, differentiate along two distinct pathways, and become either the highly invasive extravillous CTBs (EVTs) or undergo cell fusion to form the multinucleated syncytiotrophoblast (STB). ${ }^{1}$ The STB is responsible for many of the placental functions, such as transport of oxygen, nutrients and waste products, and immune tolerance. In addition, the STB is an important reproductive endocrine tissue, secreting reproductive hormones, including human chorionic gonadotrophin ( $\mathrm{hCG}$ ) and human placental lactogen. $^{2}$ The STB undergoes continuous renewal, shedding aged nuclei in the form of syncytial knots and incorporating fresh CTBs by fusion. ${ }^{2}$

Many polypeptide growth factors and hormones have been implicated in regulating trophoblast syncytium formation and placental function. For example, insulin-like growth factor (IGF)-I and its receptors ${ }^{3,4}$ and vascular endothelial growth factor (VEGF) $)^{5}$ promote trophoblast syncytialization. In contrast, transforming growth factor- $\beta$ and tumor necrosis factor- $\alpha$ inhibit trophoblast syncytialization. ${ }^{6,7}$ One common feature of these polypeptides is that their expression, maturation and activation require proteolytic processing by proprotein convertases (PCs). ${ }^{8-10}$

Of the seven known PCs, furin is of particular importance in placental development and in trophoblast fusion. We have previously reported that furin is highly expressed in human and monkey EVTs and that furin function is required for trophoblast cell invasion into the maternal endometrium. ${ }^{11}$ Furin is an essential convertase in the processing of IGF-I, type 1 IGF receptor (IGF1R) ${ }^{12,13}$ and VEGF, ${ }^{14}$ which have been implicated in trophoblast cell fusion. Most importantly, furin has been implicated in processing the envelope (Env) proteins of human endogenous retroviruses, syncytins, the only possible fusogens known to be involved in placenta syncytialization, and therefore may participate in syncytinmediated cell fusion in cells of both trophoblast ${ }^{15,16}$ and nontrophoblast lineages. ${ }^{17}$ Deletion of furin gene in mice leads to embryonic lethality at E10.5 because of the failure of

\footnotetext{
${ }^{1}$ State Key Laboratory of Reproductive Biology, Institute of Zoology, Chinese Academy of Sciences, Beijing 100101, China; ${ }^{2}$ Graduate School of the Chinese Academy of Sciences, Beijing 100039, China and ${ }^{3}$ Laboratory Animal Center, Chongqing Medical University, Chongqing, China

${ }^{*}$ Corresponding author: H Wang, Institute of Zoology, Chinese Academy of Sciences, Beichen West Road, Chaoyang district, Beijing 100101, China. Tel: +86 10 64807187; Fax: +86 10 64807187; E-mail: wanghm@ioz.ac.cn

or H-Y Lin, Institute of Zoology, Chinese Academy of Sciences, Beichen West Road, Chaoyang district, Beijing 100101, China. Tel: +86 10 64807189; Fax: + 8610 64807189; E-mail: linhy@ioz.ac.cn

Keywords: placenta; placenta-specific knockdown; furin; syncytialization; syncytiotrophoblast

Abbreviations: CK7, cytokeratin 7; CTBs, cytotrophoblast cells; EVTs, extravillous cytotrophoblast cells; FSK, forskolin; hCG, human chorionic gonadotrophin; ICM, inner cell mass; IGF, insulin-like growth factor; IGF1R, type 1 IGF receptor; PACE4, paired basic amino-acid-cleaving enzyme 4; PCs, proprotein convertases; PE, pre-eclampsia; STB, syncytiotrophoblast; TE, trophectoderm; VEGF, vascular endothelial growth factor

Received 17.9.12; revised 02.2.13; accepted 28.2.13; Edited by Y Shi
} 
allantois-chorion fusion (a key step during mouse placental trophoblast differentiation and a prerequisite for the following syncytialization), cardiac ventral closure defects and hemodynamic insufficiency. ${ }^{18}$ Because furin is expressed in both placental and embryonic tissues, ${ }^{18}$ this complex phenotype likely reflects the loss of both placental and embryonic furin.

In this study, we have employed multiple experimental models, including choriocarcinoma BeWo cells, primary human CTBs and placental explants, as well as lentivirusmediated gene targeting in mouse TE, to study the function of furin in placental trophoblast differentiation. Herein, we demonstrate that furin is highly expressed during trophoblast syncytialization and that inhibition of furin, either by siRNAs or by inhibitors, diminishes trophoblast cell fusion in vitro, prevents trophoblast syncytialization in situ and blocks normal embryonic development.

\section{Results}

Furin activity is required for BeWo cell fusion in vitro. We first determined if furin was involved in trophoblast cell fusion using the choriocarcinoma BeWo cell model. Treatment of BeWo cells with forskolin (FSK) caused timedependent upregulation of $\beta$-hCG expression, a biomarker of trophoblast cell fusion. ${ }^{17}$ Indeed, FSK treatment of BeWo cells also increased furin expression, in a time course similar to that of $\beta$-hCG expression (Figure 1a). In contrast to furin, two other ubiquitously expressed PCs, PC5 and PC7, were not altered in BeWo cells during FSK treatment (Figure 1b).

To determine if furin expression is required for cell fusion, we transfected BeWo cells with control siRNA or furin-specific siRNA for $48 \mathrm{~h}$, and then treated the cells with FSK for an additional $24 \mathrm{~h}$. While control siRNA did not affect

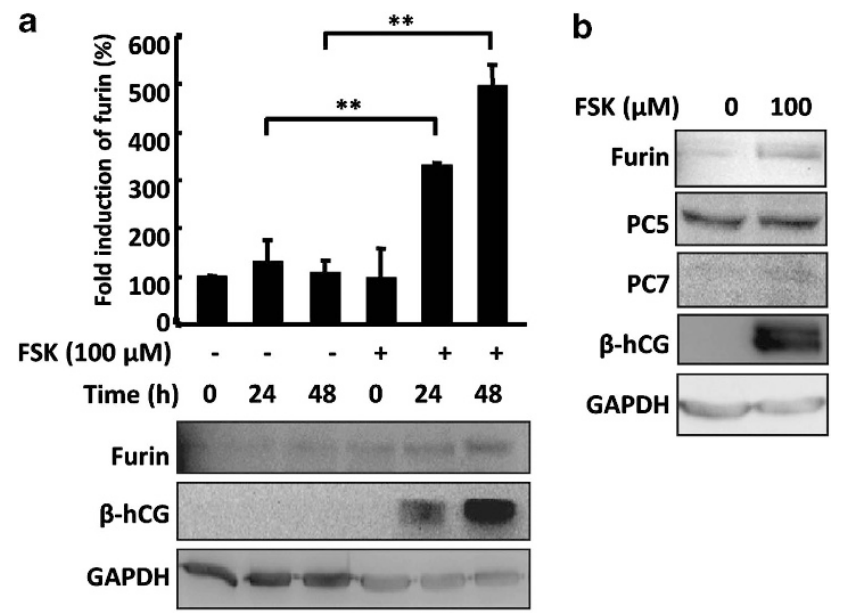

Figure 1 Increase of furin expression during FSK-induced syncytialization of choriocarcinoma BeWo cells. (a) BeWo cells were incubated in the medium with or without $100 \mu \mathrm{M}$ FSK (methanol as a vehicle control, also hereafter) for the indicated time before being subjected to protein extraction and western blotting with the indicated antibodies. Shown at the bottom are representative western blotting images. Three independent experiments were quantified by measuring the intensities of furin protein bands relative to glyceraldehyde 3-phosphate dehydrogenase (GAPDH) controls (top) $\left({ }^{* *} P<0.01\right.$; analysis of variance (ANOVA)). (b) BeWo cells treated with or without $100 \mu \mathrm{M}$ FSK for $48 \mathrm{~h}$ were lysed and the resulting extracts were subjected to western blotting with the indicated antibodies
FSK-induced expression of $\beta$-hCG (Figure 2a) or cell fusion (Figure $2 \mathrm{~b}$, right panel), furin siRNA decreased both $\beta$-hCG expression (Figure 2a) and cell fusion (Figure 2b, right panel).

As an alternative approach to interfere with furin function, we employed two inhibitors, decRVKR-CMK and $\alpha 1-P D X$. DecRVKR-CMK is a potent peptide inhibitor of all PCs and $\alpha 1$-PDX, derived from $\alpha 1$-antitrypsin, is a highly selective inhibitor of furin. ${ }^{19}$ Treating BeWo cells with decRVKR-CMK significantly reduced cell fusion (Figure 2c, right panel), as well as $\beta$-hCG expression (Figure 2c, upper left panel). Transfection of $\mathrm{pRc} / \mathrm{CMV}-\alpha 1-\mathrm{PDX}$, but not $\mathrm{pRc} / \mathrm{CMV}$ empty vector, significantly reduced $\beta$-hCG expression (Figure $2 \mathrm{c}$, lower left panel). On the other hand, transfecting BeWo cells with PC7 siRNA diminished PC7 expression but did not affect cell fusion or $\beta$-hCG expression (Figure $2 \mathrm{~d}$ ).

These results suggest that furin activity is required for syncytialization of BeWo cells in vitro. To further demonstrate this, we examined the processing of human IGF1R in BeWo cells. We chose IGF1R for two reasons. First, pro-IGF1R is a well-known furin substrate and the generation of mature IGF1R in vivo requires furin activity. ${ }^{20}$ Second, IGF1R is thought to play an important role in trophoblast cell syncytialization. ${ }^{21}$ Indeed, FSK treatment increased the levels of mature IGF1R $\beta$ (Figure 2e) in a time- and concentrationdependent manner. In primary human term CTBs transfected with furin siRNA, substantial IGF1R $\beta$ remained as pro-IGF1R $(200 \mathrm{kDa})$, corresponding to diminished expression of furin and $\beta$-hCG (Figure 2f). Similarly, administration of decRVKRCMK decreased the level of mature IGF1R $\beta$, and increased its precursor in a dose-dependent manner in primary human term CTBs (Figure 2g).

Furin is required for trophoblast syncytialization in situ. To further assess the role of furin in trophoblast syncytialization, we employed human placental tissues, donated with informed consent from patients in Beijing Xuan Wu Hospital. In the first approach, we isolated CTBs from term placentas, which are CK7 positive (Supplementary Figure 1), and cultured them in vitro for various times before cell lysis and immunoblotting. As shown in Figure 3a, these CTBs exhibited a gradual increase of $\beta$-hCG expression, most prominently in cultures after $48 \mathrm{~h}$. Interestingly, furin expression was increased during in vitro culture, attaining similar high levels at $48 \mathrm{~h}$. Immunofluorescence microscopy indicated that at the beginning $(0 \mathrm{~h})$ these CTBs were all mononucleated, but at $48 \mathrm{~h}$ following in vitro culture, patches of multinucleated cells (Figure 3b, white star) were observed among mononucleated cells. Furthermore, furin expression was most evident in multinucleated cells (Figure 3b).

In a second approach (Supplementary Figure 2), we employed the placental explant model, developed by Baczyk et al., ${ }^{22}$ to further examine the role of furin in trophoblast syncytialization. We first carried out immunofluorescence microscopy to determine the localization of furin in human placental villi at first trimester. Furin was coexpressed with $\beta$-hCG in STB, whereas neither furin nor $\beta$-hCG was appreciably expressed in CTBs (Figure 4A). We then collected placental villi from 8- to 12-week-old placentas and cultured them in transwell. Villi were subjected to limited trypsin treatment to remove selectively the syncytial layer. 

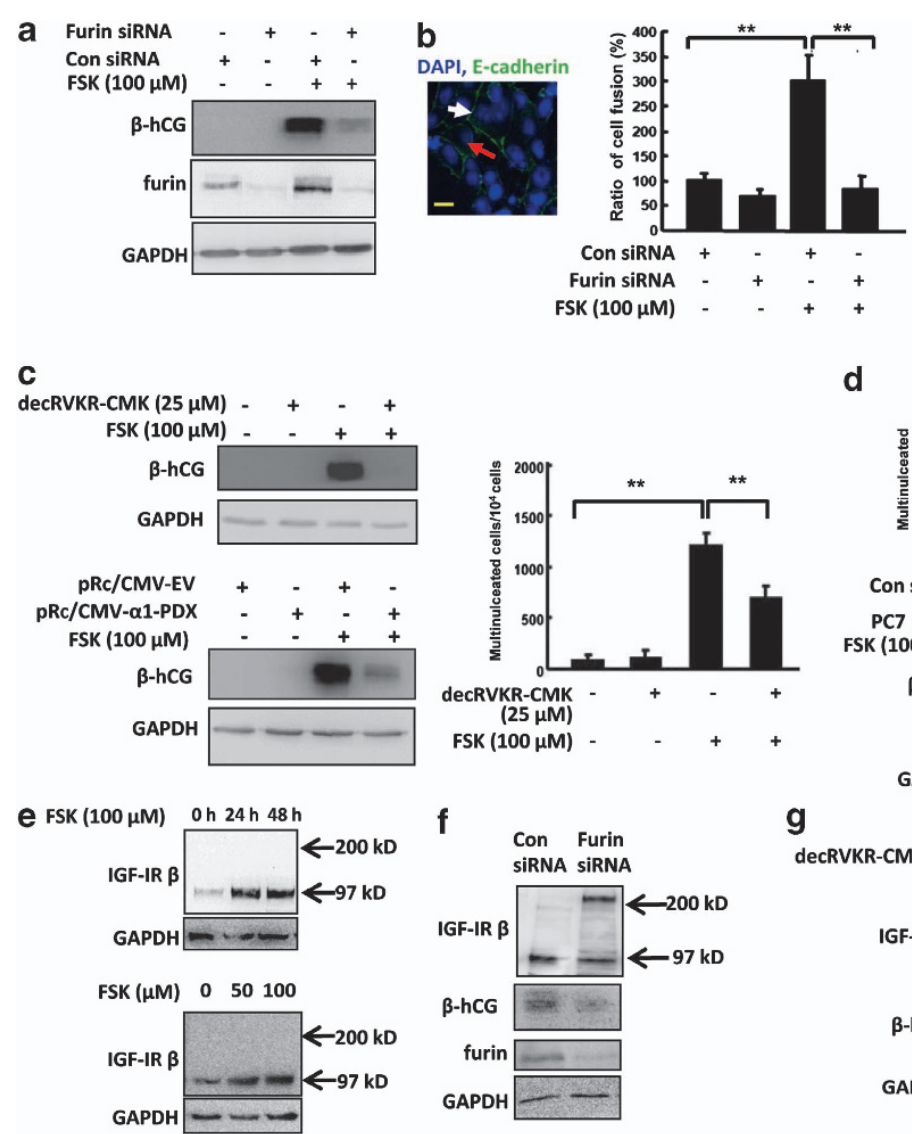

d
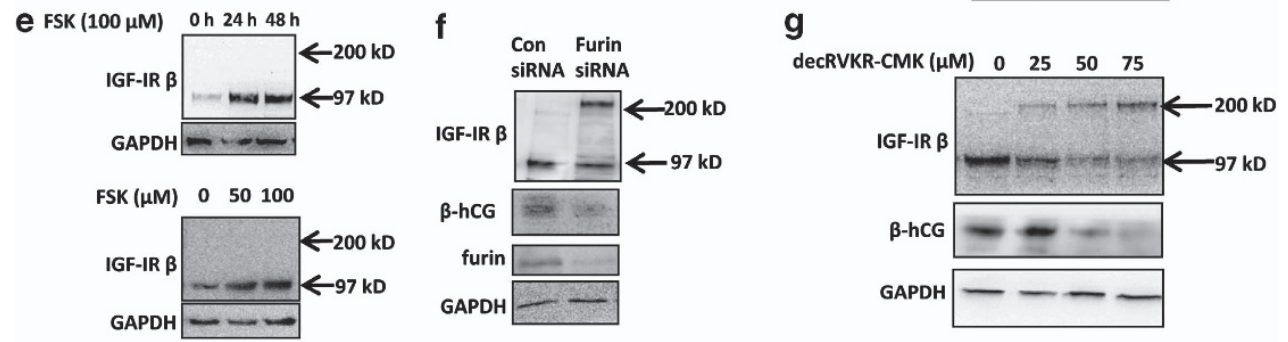

Figure 2 Furin is required for FSK-induced syncytialization of choriocarcinoma BeWo cells. (a and b) BeWo cells treated with control (Con) small interfering RNA (siRNA) or furin siRNA were incubated in the medium with or without FSK for $48 \mathrm{~h}$. (a) Cells were lysed and the resulting extracts were subjected to western blotting with the indicated antibodies. (b) Cells were stained with 4',6-diamidino-2-phenylindole (DAPI) (blue) and anti-E-cadherin (green). Shown is a typical image of mononucleated (red arrow) or multinucleated (white arrow) cells (left panel). Bar $=20 \mu \mathrm{m}$. Three independent experiments were quantified by analyzing the ratio of multinucleated cells in different treatment groups (right panel) $\left({ }^{* *} P<0.01\right.$; analysis of variance (ANOVA)). (c) BeWo cells were treated with decRVKR-CMK (dimethyl sulfoxide (DMSO) as its vehicle control) or transfected with $\mathrm{pRc} / \mathrm{CMV}-\alpha 1-\mathrm{PDX}$ (pRc/CMV-EV as an empty vector control) for $24 \mathrm{~h}$, followed by treatment with $100 \mu \mathrm{M}$ FSK for $48 \mathrm{~h}$. Cells were lysed and extracts were immunoblotted by the indicated antibodies. The right panel summarizes three independent experiments on the effect of decRVKR-CMK pretreatment on FSK-induced BeWo cell syncytialization ( ${ }^{* *} P<0.01$; ANOVA). (d) BeWo cells were transfected with control siRNA or PC7 siRNA for $24 \mathrm{~h}$, followed by treatment with or without FSK for $48 \mathrm{~h}$. Cells were harvested for multinucleated cell counting (upper panel) and the lysed extracts were subjected to western blotting with the indicated antibodies (lower panel). (e) Levels of IGF1R $\beta(97 \mathrm{kDa})$ in BeWo cells treated with FSK for the indicated time (upper panel) and concentration (lower panel). Experiments were repeated three times and the representative data are shown. (f) Primary cultured CTBs were transfected with furin siRNA or control (Con) siRNA for $48 \mathrm{~h}$. Proteins were extracted and subjected to western blotting using indicated antibodies. The pro-IGF1R $\beta(200 \mathrm{kDa})$ and its mature form $(97 \mathrm{kDa})$ were shown. (g) Primary cultured CTBs were treated with indicated concentrations of decRVKR-CMK for $48 \mathrm{~h}$ before being subjected to protein extraction and western blotting using indicated antibodies. CTBs used in (f) and (g) were isolated from three normal term placentas, and the representative data are shown

The 'denuded' villi regenerated their STB layer from the CTBs lining the villi, when cultured in $8 \% \mathrm{O}_{2}$, thought to be the physiological placental oxygen tension in early pregnancy. ${ }^{1}$ Hardly detectable levels of $\beta$-hCG staining could be seen after trypsin treatment, indicating the efficiency of STB removal (Figure 4Bb). Following $48 \mathrm{~h}$ of incubation, a complete layer of syncytium, positive for $\beta$-hCG and with multiple nuclei, was observed overlaying $\beta$-hCG-negative mononucleated CTBs (Figure 4Bc). Treating the denuded villi with control siRNA did not affect the reformation of STB (Figures $4 \mathrm{C}$ a and e). In contrast, furin siRNA completely abolished STB reformation (Figures $4 \mathrm{C} \mathrm{b}$, fand $\mathrm{D}, P<0.01$ ). Addition of FSK to the culture medium caused more robust syncytialization, as indicated by overcrowding of nuclei in the thicker $\beta$-hCG-positive STB layer (Figures $4 \mathrm{Cc}$ and $\mathrm{g}$ ), but this again was abolished by furin
siRNA (Figures $4 \mathrm{Cd}, \mathrm{h}$ and $\mathrm{D}, P<0.01$ ). Cotransfecting the placental villi with furin siRNA and an FITC-tagged siRNA confirmed the uniform transfection (Figure 4E). Furthermore, immunoblotting of placental villi extracts confirmed that furin siRNA efficiently inhibited furin expression (Figure 4F). Similarly, addition of a PC inhibitor decRVKR-CMK also inhibited both 'spontaneous' and FSK-enhanced reformation of syncytial layer (Figure 4G).

To determine whether furin is dysregulated under certain pathological conditions, we examined the expression of furin in the placentas from patients of pre-eclampsia (PE). Similar as in first trimester placentas (Figure 4Aa), furin was also mainly expressed in the STB in term placentas (Figure $4 \mathrm{H}$ ). However, term placentas of PE patients exhibited more 'patchy' furin expression in the STB compared with their 
gestational age-matched controls (Figure 4H). To further quantify the levels of furin expression in the above two groups, we analyzed multiple placentas of each group by immunoblotting of placental villi extracts. These experiments indicated that PE placental villi expressed significantly reduced furin compared with their control counterparts $(P<0.01$; Figure $4 \mathrm{I})$.

\section{Placenta-specific knockdown of furin in the mouse leads} to severe defects in syncytialization and embryonic lethality. The above results clearly suggest that furin plays an essential role in trophoblast syncytialization in human placentas. To gain further physiological insight into furin function in mammalian placentation and pregnancy, we utilized the mouse model. First, we determined that furin mRNA was highly expressed in the mouse placental spongiotrophoblast and labyrinth layers at E9.5-10.5

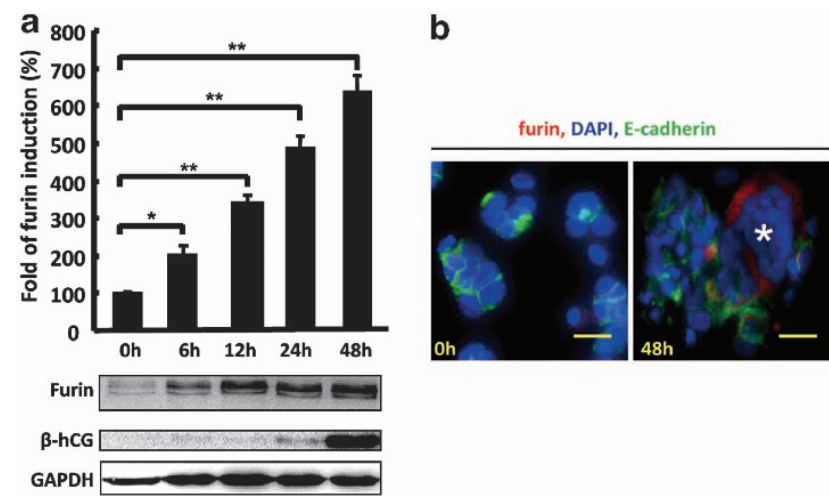

Figure 3 Increase of furin expression during spontaneous syncytialization of primary cultured human term CTBs. (a) CTBs were cultured in vitro for indicated time before being subjected to protein extraction and western blotting with the indicated antibodies (bottom). Three parallel experiments were quantified by measuring the intensities of furin protein bands relative to glyceraldehyde 3-phosphate dehydrogenase (GAPDH) controls (top; ${ }^{*} P<0.05$; ${ }^{* *} P<0.01$; analysis of variance (ANOVA)). (b) CTBs were fixed and subjected to immunofluorescence staining using anti-E-cadherin (green) and anti-furin (red) antibodies. CTBs were isolated from five normal individual placentas, and the representative images are shown. White stars depict typical multinucleated cells, and blue depicts 4',6-diamidino-2-phenylindole (DAPI) staining. The two images are of the same magnification. $\mathrm{Bar}=20 \mu \mathrm{m}$
(Supplementary Figure 3A). To interfere specifically with furin expression in the placenta, we employed the lentivirusmediated RNAi method developed by Okada et al. ${ }^{23} \mathrm{We}$ produced lentiviruses carrying shRNA for furin (Supplementary Figures $3 \mathrm{~B}$ and $\mathrm{C}$ ) and infected blastocyststage embryos with these viruses, along with lentiviruses expressing EGFP. After $8 \mathrm{~h}$ of incubation, the embryos were examined and the TE but not the inner cell mass (ICM) of these embryos exhibited green fluorescence (Figure 5A). Downregulation of furin protein by the furin shRNA was also confirmed (Figure 5D). The embryos were implanted into pseudopregnant recipient mice, followed by euthanization during E8.5-E13.5 for examination. In all, 74.0 and $58.6 \%$ of embryos in the furin shRNA group were degenerate at E12.5 (Figure 5B and Table 1) and E13.5 (Table 1), respectively $(P<0.01$ as compared with the control group). In contrast, most embryos in the control shRNA group appeared normal (Table 1 and Figure 5B). Among the live fetuses at these stages in the furin knockdown group, the placentas were significantly smaller than those of the control shRNA group (Figure 5C).

In all cases, the placentas, but not the embryos, were EGFP positive as observed under fluorescence anatomical lens (Figure 5C, representative images from E12.5 and Supplementary Figure 3D), indicating that lentivirus-mediated EGFP (and furin shRNA) expression remained restricted to placental tissues at these mid-gestation stages.

In situ hybridization analyses indicated that placentaspecific knocking down of furin had no obvious effect on the development of spongiotrophoblast (Figure 5E), but significantly suppressed branching of labyrinth, resulting in a much thinner labyrinth layer, based on the localization of Tpbp mRNA, a marker of spongiotrophoblast layer (Figure 5E), and Gcm1 mRNA, a marker for the labyrinth layer (Supplementary Figure 3E). Furthermore, electron microscopy (EM) study showed that four-layered structure in both control and furin shRNA-treated placentas are present (Figure 5F), but the structure of furin shRNA-treated placentas became disruptive, including poor interactions between STBs, ST-I and ST-II (Figure 5Fd), unfused ST-II layer (Figure 5Fe) and decreased lipid drops in ST-II layer (Figures $5 \mathrm{Fa}$ and $\mathrm{c}$ ).

Figure 4 Furin is required for STB formation in human placental explants. (A) Immunofluorescence images of the first trimester $(n=9)$ human placental villi. Green, furin; red, $\beta$-hCG; blue, 4',6-diamidino-2-phenylindole DAPI. Control is an image using a same concentration of normal rabbit serum in place of first antibodies. All the images are of the same magnification as shown in the control image. Bar $=50 \mu \mathrm{m}$. (B) Representative images of human placental explant denudation model. (a) Untreated placental explant showing a single layer of CTB (white arrows) and a healthy layer of STB (orange arrows). (b) Placental explant after gentle trypsinization and subsequent 24-h culture. (c) Spontaneous reformation of new STB after $72 \mathrm{~h}$ in culture. Red, $\beta$-hCG; green, CK7; blue, DAPI. ST, stromal cells. Bar $=50 \mu$ m. (C) Denuded villi were cultured for $72 \mathrm{~h}$ in the presence of control (Con) small interfering RNA (siRNA) (a and $e$ ), furin siRNA ( $b$ and $f$ ), control siRNA plus FSK ( $c$ and $g$ ) or furin siRNA plus FSK ( $d$ and $h$ ). The villi were then embedded, sectioned and immunostained with antibodies against $\beta$-hCG (red) and with DAPI (blue). (a), (b), (c) and (d) depict overall images with representative areas (rectangles) magnified in $(\mathrm{e}),(\mathrm{f}),(\mathrm{g})$ and $(\mathrm{h})$, respectively. All the images in the same row are of the same magnification. Bar $=100 \mu \mathrm{m}$. Green lines indicate the thickness of the STB layer. (D) Three independent experiments in (C) were quantified by analyzing the STB regeneration rate, which is calculated as the percentage of the number of nuclei in $\beta$-hCG-positive STB layer divided by the total number of nuclei in CTB cells and STB ( ${ }^{*} P<0.01$; analysis of variance (ANOVA)). (E) Cotransfection of the placental explants with furin siRNA and a fluorescein isothiocyanate (FITC)-tagged siRNA. (F) Denuded villi treated in (C) were subjected to protein extraction and immunoblotting analyses. Shown on the left are representative blots and on the right is a summary of three independent experiments $\left({ }^{* *} P<0.01\right.$; ANOVA). (G) Denuded villi were cultured for $72 \mathrm{~h}$ in the presence of a PC inhibitor decRVKR-CMK and/or FSK. Statistical analysis of STB regeneration rates of three independent experiments is shown (** $P<0.01$; ANOVA). (H) Representative images of immunofluorescence of term placental villi from control patients $(n=12)$ and PE patients $(n=12)$. Green, furin; blue, DAPI. Bar $=50 \mu \mathrm{m}$. (I) Total placental proteins were extracted from individual placentas as in $\mathbf{( H )}$ and analyzed by immunoblotting with the indicated antibodies. Representative blots are shown. Normal, control patients; PE, pre-eclampsia. ${ }^{* \star} P<0.01$ 


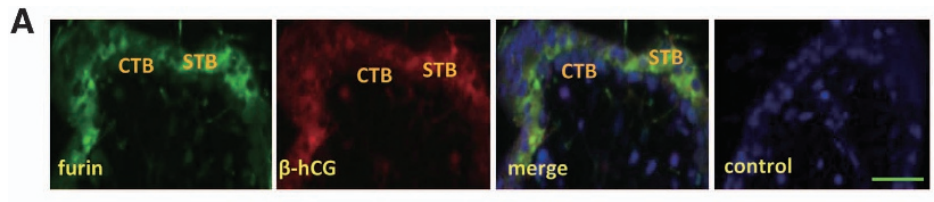

B

$\beta$-hCG, DAPI, CK7

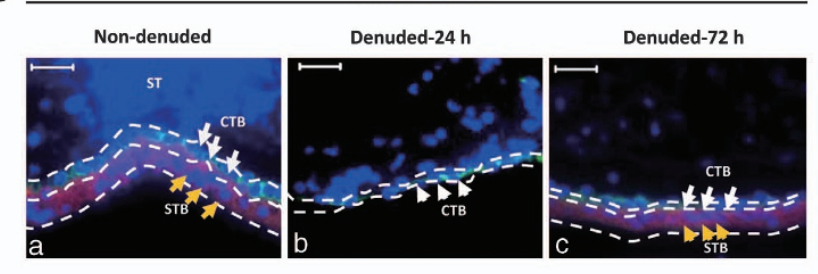

C

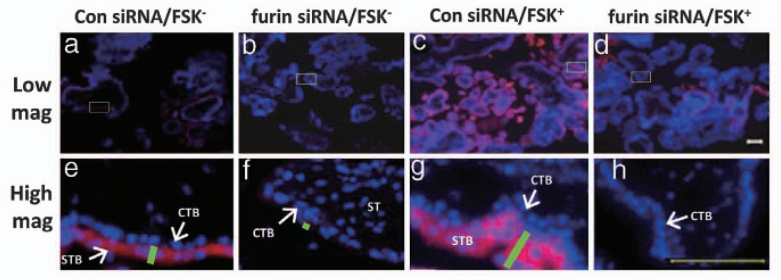

D

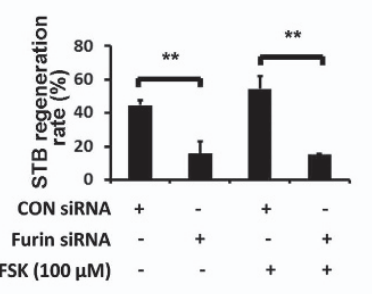

$\mathbf{F}$

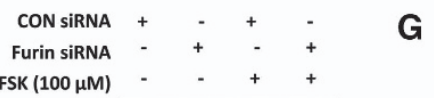

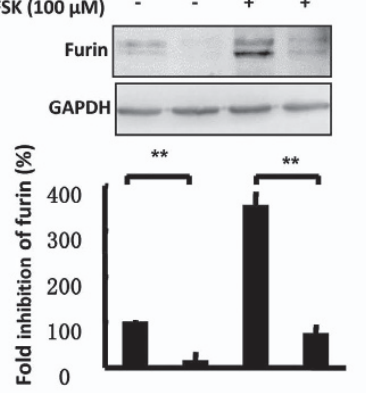

E
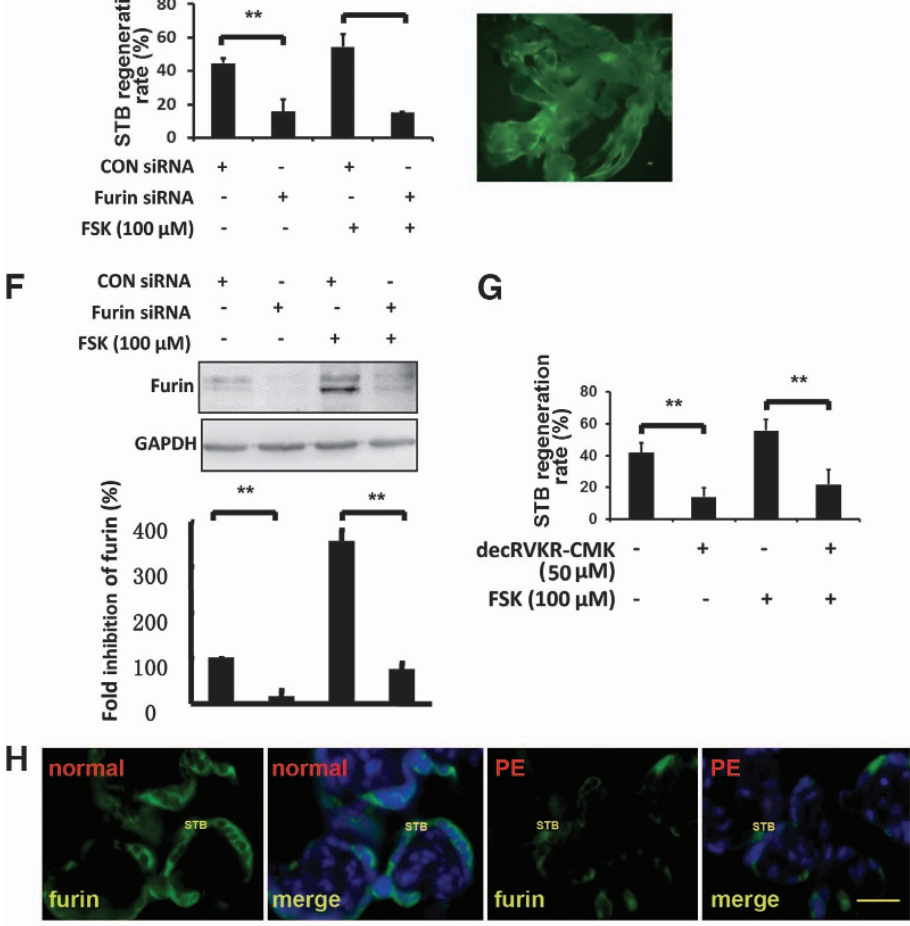

I
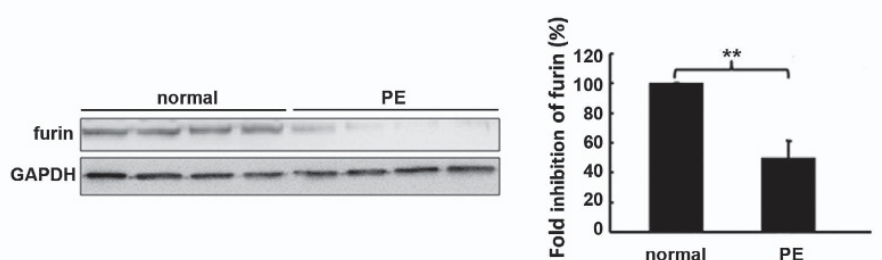
A

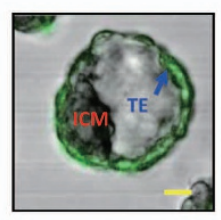

B

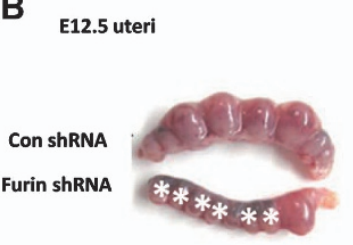

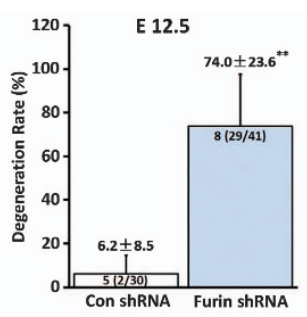

E
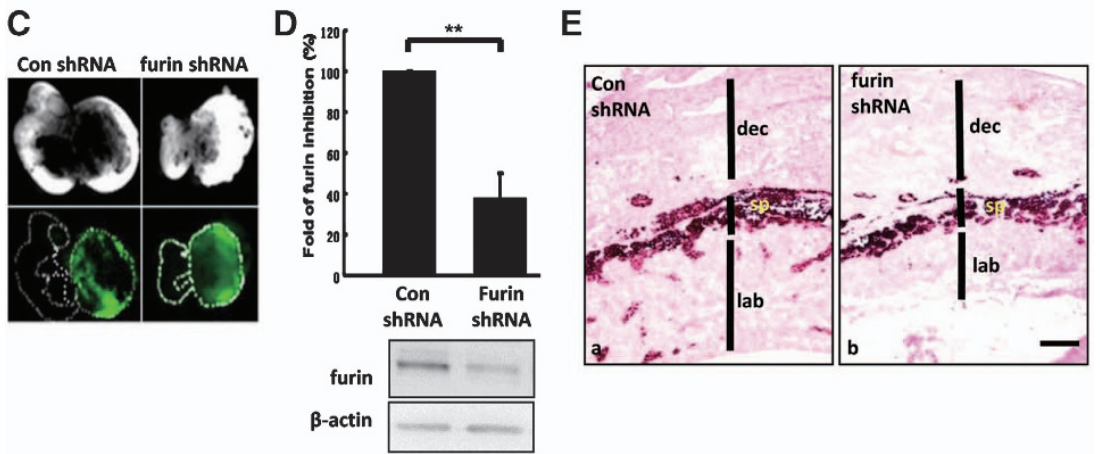

$\mathbf{F}$
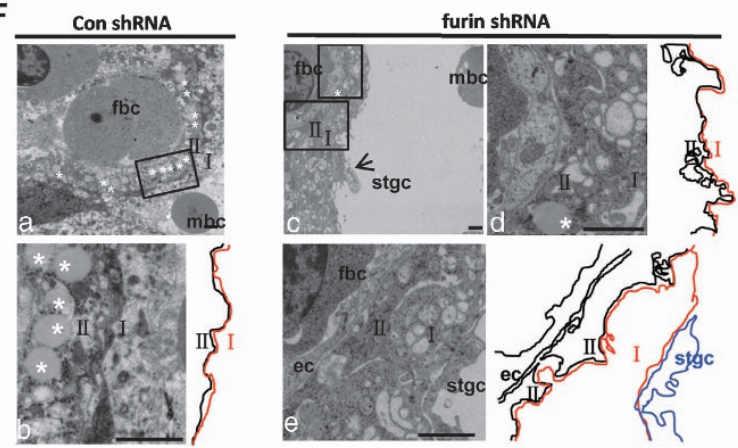

Figure 5 Placenta-specific knockdown of furin causes severe placental syncytialization defects in mice. (A) A fluorescence anatomical view of a zona-free blastocyst embryo, which has been incubated for $8 \mathrm{~h}$ with $7 \times 10^{6} \mathrm{TU} / \mathrm{ml}$ lentivirus-carrying furin shRNA and green fluorescent protein (GFP), followed by further incubation for $40 \mathrm{~h}$ to allow GFP protein expression. Green, GFP. Bar $=20 \mu \mathrm{m}$. (B, left panel) Typical images of two uterine horns carrying furin shRNA- or control (Con) shRNA-treated embryos at E12.5. White stars indicate degenerate embryos; (right panel) a statistical analysis of the ratio of degenerate embryos at E12.5 ( ${ }^{* *} P<0.01 ; t$-test). Numbers within the bars are: the number of pregnant mice (the number of degenerate embryos/the number of implantation sites). (C) Representative images of placentas and embryos dissected out from the uterine horns in (B) and photographed by phase contrast microscope (upper panel) or observed under computer-assisted fluorescence anatomical lens (lower panel). (D) Total placental proteins were extracted from placenta samples shown in (C) and subjected to western blotting analyses, with representative blots shown at the bottom and a summary of three placentas shown at the top $\left({ }^{\star *} P<0.01\right.$; analysis of variance (ANOVA)). (E) Placentas as representatively shown in (C) were sectioned and subjected to in situ hybridization using a spongiotrophoblast marker, Tpbp. (a), Control (Con) shRNA; (b), furin shRNA. Sp, spongiotrophoblast; lab, labyrinth layer; dec, maternal decidua. $B a r=100 \mu \mathrm{m}$. (F) Four-layered interhemal barrier of the labyrinth: mononucleated sinusoidal trophoblast giant cells (stgc), the two-layered STB (ST-I and ST-II) at the maternofetal interface and the endothelial cells (ec). (a and b) Control (Con) shRNA; (c-e) furin shRNA. The ST-II-specific lipid inclusions were indicated with white stars. (b) Magnified picture of a framed region in (a). (d) and (e) The expanded views of the areas boxed in (c), with (d) showing the disruptive interactions between ST-I and ST-II, and (e) indicating the unfused ST-II cells. Outlines of the cytoplasmic membranes schematizes on the right. mbc, maternal blood cells; fbc, fetal blood cells lined by endothelial cells. Bar $=2 \mu \mathrm{m}$

Table 1 Effect of placenta-specific knockdown of furin on embryo implantation and early embryonic development

\begin{tabular}{|c|c|c|c|c|c|c|c|c|}
\hline & \multicolumn{4}{|c|}{ E12.5 } & \multicolumn{4}{|c|}{ E13.5 } \\
\hline & $\begin{array}{l}\text { No. of } \\
\text { transferred } \\
\text { embryos }\end{array}$ & $\begin{array}{l}\text { No. of } \\
\text { recipient } \\
\text { mice }\end{array}$ & $\begin{array}{l}\text { No. of } \\
\text { implantation } \\
\text { sites }\end{array}$ & $\begin{array}{l}\text { No. of degenerate } \\
\text { embryos (degenera- } \\
\left.\text { tion rate }{ }^{a}\right)\end{array}$ & $\begin{array}{l}\text { No. of } \\
\text { transferred } \\
\text { embryos }\end{array}$ & $\begin{array}{l}\text { No. of } \\
\text { recipient } \\
\text { mice }\end{array}$ & $\begin{array}{l}\text { No. of } \\
\text { implantation } \\
\text { sites }\end{array}$ & $\begin{array}{c}\text { No. of degenerate } \\
\text { embryos (degenera- } \\
\text { tion rate }{ }^{\mathrm{a}} \text { ) }\end{array}$ \\
\hline $\begin{array}{l}\text { Con } \\
\text { shRNA }\end{array}$ & 42 & 5 & 30 & $2(6.2 \pm 8.5 \%)$ & 24 & 3 & 20 & $2(9.5 \pm 16.5 \%)$ \\
\hline $\begin{array}{l}\text { Furin } \\
\text { shRNA }\end{array}$ & 64 & 8 & 41 & $29(74.0 \pm 23.6 \%)^{\star *}$ & 56 & 7 & 35 & $19(58.6 \pm 25.8 \%)^{\star \star}$ \\
\hline
\end{tabular}

${ }^{* \star} P<0.01$ as compared with the Con shRNA group.

a Degeneration rate means the ratio of degenerate embryos to the number of implantation sites and is represented as the mean \pm S.E.M. 


\section{Discussion}

In this study, we have demonstrated that furin plays an essential role in trophoblast cell fusion and syncytialization in humans. Our conclusions are based on the following lines of evidence. First, furin expression was enriched in the STB layer in first trimester human placentas. Second, in multiple models, including choriocarcinoma BeWo cells, primary human placental CTBs and human placental villi explants, trophoblast fusion and syncytialization were accompanied by upregulation of furin expression. Third, and most importantly, inhibition of furin function by multiple mechanistic strategies and experimental approaches all led to inhibition of trophoblast cell fusion and syncytialization.

We also demonstrated that in furin-deficient BeWo cells, either via siRNA-mediated inhibition of furin expression or by the administration of furin inhibitor decRVKR-CMK, significant levels of unprocessed form of the IGF1R, pro-IGF1R, accumulated. These results suggest that processing of IGF1R by furin may serve as an important mechanism for furinpromoted trophoblast syncytialization. This interpretation is consistent with previous studies implicating the IGF1 signaling system in human trophoblast cell differentiation and syncytialization, 3,4,24,25 and correlates well with the notion that IGF1R is an important physiological substrate of furin. ${ }^{11}$ Of interest, it has been previously shown that furin can process syncytin-1 and -2 into mature fusogenic proteins, ${ }^{15}$ which are the putative fusogens for syncytialization. Therefore, we found herein a possible new mechanism whereby furin promotes syncytiatlization, other than syncytin processing. Identification of other furin-specific targets during syncytialization would further help us find more important molecules or possible fusogenic proteins for syncytialization.

Human gestational diseases, including trisomy of chromosome $21^{26,27}$ and $P E,{ }^{28-30}$ have been reported to be associated with syncytialization defects. Given that furin is the only convertase, among those expressed in the placentas, exhibiting a functional involvement in cytotrophoblast cell syncytialization, we speculate that furin deficiency may be an important etiology of the above diseases. Interestingly, we found that the expression of furin was decreased in placentas from patients with PE as compared with gestational agematched normal pregnant women. It has been widely recognized that the placenta is the major contributor to the pathogenesis of this clinical complication, as delivery of the placenta remains the only available treatment. Placental hypoperfusion and ischemia, as well as placental infarcts, contribute to the pathology of PE. Moreover, disturbances of trophoblast fusion in PE placenta endanger the integrity of the placental barrier and cause the release of more syncytial knots and a systemic inflammatory response by the mother. ${ }^{28-30}$ In this regard, the fact that furin was significantly downregulated in placental tissues from PE patients suggests that low levels of furin may be associated with the onset of PE. Therefore, we need to gather data pertaining how furin was downregulated in pre-eclamptic placentas, and whether and how furin is involved in the etiology of PE.

In humans, the phenomenon of cell-cell fusion is not unique to placental tissues, but occurs in several different cell and tissue types, including the fusion between the egg and the sperm during fertilization, ${ }^{24}$ formation of multinucleated chondroclasts, osteoclasts or giant cells from mononucleated macrophages, ${ }^{25}$ fusion of skeletal muscle satellite cells with skeletal muscle fibers, ${ }^{25}$ and so on. These fusion processes share some similar mechanisms, although distinct mechanisms may be involved. Yuasa et al. ${ }^{31}$ reported that mRNA levels of PACE4 (paired basic amino-acid-cleaving enzyme 4), a member of PCs family, increased significantly during myogenesis, whereas the expression of furin and PC6 remained unchanged, highlighting a role of PACE4 in myoblast fusion. These results, together with ours, indicate that PC family members are involved in cell fusion process, and the fusionpromoting effect of PCs is likely tissue- or organ-specific.

Although the mouse placenta differs from the human placenta in its morphogenesis and endocrine functions, they do share considerable similarities, such as cell types and master regulatory molecules. ${ }^{32-34}$ Mouse STB differentiation is initiated and controlled by the bioavailability of Gcm1, a transcription factor specifically expressed in the mouse placenta. Various signaling pathways have also been reported to play critical roles in STB differentiation. ${ }^{32,34}$ Furin was reported to be highly expressed in chorion and allantois during embryo development. ${ }^{18}$ Furin-null mice died at E8.5E10.5 because of a failure in the chorio-allantoic fusion, which starts from E8.0 and develops into the chorion plate (E8.5) and labyrinth layer (E10.0) afterwards. ${ }^{18}$ Syncytialization initiates at the tips of elongating branches of the chorion plate and ultimately forms two-layered STB, SynT-I and SynT-II, in the labyrinth layer. ${ }^{34}$ In this study, furin mRNA was found to be extensively distributed in the labyrinth layer and the spongiotrophoblast layer of the mouse placenta (data not shown). The phenotypic defects in furin-null mice preclude the possibility to explore the physiological significance of furin on later placental events, such as trophoblast fusion, employing the systemic null mutant mice. Lentivirus-mediated placentaspecific gene manipulation strategy uses lentiviral infection of mouse blastocysts before implantation and consequently the infection is restricted to the placenta and not the fetus. ${ }^{23,35}$ It allows placenta-specific gene manipulation and can be utilized to rescue the embryonic lethal phenotypes of gene knockout mice caused by placental defects. ${ }^{24}$ More recently, this strategy was utilized to generate a placenta-specific soluble fms-like tyrosine kinase-1-overexpressed mouse model, which phenocopies the symptoms of $P E,{ }^{36}$ suggesting that this strategy could better trace pathological events during the onset of pregnancy-related diseases, as in most cases the decrease or increase of (instead of completely lost of) certain genes at certain stages of pregnancy leads to complications such as PE and intrauterine growth restriction. Our results showed that lentivirus-driven specific knockdown of furin in the mouse placenta led to severe embryonic lethality, and that furin knockdown placenta showed a thinner labyrinth as compared with the control placenta. Further studies using EM showed disruptive STBs in the labyrinth layer. Guzman-Ayala et al. $^{37}$ have reported that loss of function of furin in early mouse embryos leads to an upregulation of mammalian achaete/scute homolog $2,,^{37}$ a transcription factor that prevents trophoblast differentiation along the STB pathway. ${ }^{38}$ Taken together, these data suggest that furin is also indispensable for the syncytialization of the mouse placenta. 
In summary, our results suggest a novel function of furin as a promoter for syncytialization in both humans and mice, and also help to shed light on the roles of PC family members during various types of cell fusion.

\begin{abstract}
Materials and Methods
Placental tissue collection. Human placental tissues from mothers aged 25-40 years were collected in accordance with the policy of Ethics Committees of Xuan Wu Hospital and Chinese PLA General Hospital in Beijing. Informed consent was obtained from each woman donating her placenta. In all cases, discarded placenta samples were anonymized, and informed consent was obtained from each woman donating her placenta. This study and the use of samples were under standard experimental protocols approved by the Ethics Committee of the Institute of Zoology, Chinese Academy of Sciences. For immunostaining and western blotting, 21 normal human placentas at different gestational stages ( 9 at the first trimester and 12 at term) were collected from women undergoing legal abortion or normal delivery. In all, 12 placentas from late-onset pre-eclamptic patients whose gestational ages matched with normal controls were also collected (term deliveries at 37 to 40 weeks). PE was defined as new onset of hypertension (systolic/diastolic blood pressure higher than $140 / 90 \mathrm{~mm} \mathrm{Hg}$ ) measured on two occasions of at least $4 \mathrm{~h}$ apart, and proteinuria (excretion of more than $300 \mathrm{mg}$ of urinary protein per $24 \mathrm{~h}$ ) after 20 weeks of gestation. The time of the clinical onset of PE was defined when both hypertension and proteinuria occurred. None of the pre-eclamptic patients involved in this study were complicated with obesity, chronic hypertension, gestational diabetes mellitus or eclampsia. All tissues were fixed in $4 \%$ paraformaldehyde (PFA), followed by paraffin embedding. For syncytial denudation and spontaneous syncytialization studies, eight placental villi at 8-12 weeks of gestation and five placentas at term delivered by cesarean section were collected, respectively, under sterile condition.
\end{abstract}

BeWo cell culture and fusion assay. The human choriocarcinoma cell line BeWo was maintained in Ham's F-12 medium supplemented with $15 \%$ fetal bovine serum (FBS; Gibco BRL, Carlsbad, CA, USA), 100 U/ml penicillin and $100 \mu \mathrm{g} / \mathrm{ml}$ streptomycin in $5 \% \mathrm{CO}_{2} / 95 \%$ air at $37^{\circ} \mathrm{C}$. Cell fusion was induced by treating BeWo cell with FSK (Sigma-Aldrich, St. Louis, MO, USA). ${ }^{22}$ Cell fusion was assessed by three independent methods: (1) immunofluorescence (see below) using anti-human E-cadherin antibody (sc-71008; Santa Cruz Biotechnology, Santa Cruz, CA, USA) to show the loss of cell membrane during the fusion process. The number of multinucleated cells in five randomly selected areas under the microscope was counted. (2) BeWo cells were stained with propidium iodide (PI; Sigma) and analyzed by flow cytometry to quantify polyploid cells. (3) Wholecell lysates of BeWo cells subjected to cell fusion induction exhibited increased the level of $\beta$-hCG by western blot (see below).

Syncytial denudation and reformation of STB. Syncytial denudation was performed as described previously.2 Briefly, individual clumps of placental villi at 8-12 weeks of gestation were dissected in sterile cold PBS. Under a dissecting microscope, the proximal stems of individual clumps of villi were grasped using fine dissecting forceps to push the stem into the underside of a sterile polystyrene cube (Costar, Cambridge, MA, USA). Explanted clumps were transferred individually to tissue culture wells containing $0.125 \%$ trypsin (Sigma) for $5 \mathrm{~min}$ at $37^{\circ} \mathrm{C}$ to denude the STB layer. Trypsin digestion was terminated by washing two timesin PBS containing $10 \% \mathrm{FBS}$. Individual cubes with villi were transferred to the culture well containing serum-free Dulbecco's modified Eagle's medium (DMEM)/F-12 medium (Gibco, Grand Island, NY, USA), 1\% liquid media supplemented with ITS +1 (Sigma), $100 \mathrm{U} / \mathrm{ml}$ penicillin, $100 \mathrm{U} / \mathrm{ml}$ streptomycin, $2 \mathrm{mM}$ L-glutamine and $100 \mathrm{mg} /$ $\mathrm{ml}$ gentamicin) with furin siRNA $(100 \mathrm{nM})$, non-silencing siRNA control or furin inhibitor $(50 \mu \mathrm{M})$ administrated in the presence or absence of $100 \mu \mathrm{M}$ FSK under $8 \%$ ambient oxygen $(40 \mathrm{~mm} \mathrm{Hg})$ condition. Each experiment employed villi isolated from the same placenta to ensure sample uniformity. Explants were fixed and waxembedded to assess morphology at $\mathrm{Oh}$ and throughout the duration of the experiments (24 and $72 \mathrm{~h}$ ). Tissue sections were immunostained with $\beta$-hCG to assess the regeneration of STB and with DAPI (blue) to mark the nuclei. The regeneration rate is calculated as the percentage of the number of nuclei in $\beta$-hCGpositive STB layer divided by the total number of nuclei in CTB cells and STB.

Primary term cytotrophoblast culture and spontaneous syncytialization. Isolation of CTBs from the human term placenta was performed as described previously. ${ }^{39}$ Briefly, the placenta was cut into pieces and digested in DMEM with $0.125 \%$ trypsin and $0.03 \%$ DNase-I (Sigma). CTBs were separated by percoll (GE Healthcare, Bio-sciences AB, Uppsala, Sweden) density gradient centrifugation. More than $95 \%$ of the cells harvested are CTBs as indicated by positive staining of cytokeratin 7 (CK7), a marker for CTBs. Cells $\left(2 \times 10^{6}\right.$ per well) were plated in 6-well plates in DMEM with $10 \% \mathrm{FBS}, 100 \mathrm{U} / \mathrm{ml}$ penicillin and $100 \mu \mathrm{g} / \mathrm{ml}$ streptomycin, and then cultured in $5 \% \mathrm{CO}_{2} / 95 \%$ air incubator at $37^{\circ} \mathrm{C}$. CTBs spontaneously fused to form syncytium in vitro as confirmed by immunostaining of fixed cells with E-cadherin, followed by counting of multinucleated syncytium or flow cytometry to determine the number of polyploid cells, or the increase in the secretion of $\beta$-hCG.

Animals. All animal use in this study was approved by the Animal Care and Use Committee of the Institute of Zoology, Chinese Academy of Sciences. To prepare placental tissues for in situ hybridization (ISH), female and male CD1 mice were housed overnight. The day when vaginal plug was first observed was defined as E0.5. Mice were killed at E9.5-E14.5, and placentas were collected and frozen into $-80^{\circ} \mathrm{C}$ for cryosection. Animals used to prepare for the placenta-specific furin knockdown study was detailed in 'Generation of placenta-specific furin knockdown mice'.

ISH of mouse placentas. ISH was performed using digoxigenin (DIG)labeled probes (furin and Tpbp) or ${ }^{35} \mathrm{~S}$-labeled probes (Gcm1) on $10-\mu \mathrm{m}$ frozen sections of mouse placentas. ${ }^{40}$ Sections were fixed in ice-cold $4 \%$ PFA in PBS for $60 \mathrm{~min}$. Prehybridization was carried out in a humidified chamber at room temperature in $5 \times$ SSC (standard saline citrate), $50 \%$ deionized formamide, $1 \times$ Denhardt's reagent, $10 \%$ dextran sulfate and salmon sperm DNA (100 $\mu \mathrm{g} / \mathrm{ml})$. Hybridizations with DIG-labeled CRNA probes were carried out at $55^{\circ} \mathrm{C}$ overnight. Slides were washed in $2 \times$ SSC at room temperature for $30 \mathrm{~min}$, followed by treatment with RNase-A $(100 \mathrm{ng} / \mathrm{ml})$ and additional washes with $2 \times \mathrm{SSC}$ for $30 \mathrm{~min}$ at room temperature, $2 \times \mathrm{SSC}$ for $1 \mathrm{~h}$ and $0.1 \times \mathrm{SSC}$ for $1 \mathrm{~h}$ at $55^{\circ} \mathrm{C}$. Hybridized probes were detected using an alkaline phosphatase-conjugated antiDIG Fab fragment (1:2000; Roche Diagnostics GmbH, Mannheim, Germany) and 5-bromo-4-chloro-3-indolyl phosphate/Nitro blue tetrazolium chloride (Promega Co., Madison, WI, USA). For Gcm1, hybrids were detected after 3 weeks of autoradiography using Kodak NTB-2 liquid emulsion. Hybridization with sense probes was included as controls for nonspecific hybridization, and no positive staining was found with the sense probes.

Generation of placenta-specific furin knockdown mice. The vector, pLL3.7, modified from pBFGW plasmid, contains a CMV promoter driving EGFP and a mouse U6 promoter driving shRNAs. ${ }^{41}$ Viral particles were packaged as reported previously. ${ }^{23}$ In brief, furin shRNA (top strand: $5^{\prime}$-TCACGGATG ATCTCCACATCATTCTCTTCAAGAGAGAGAATGATGTGGAGATCATCCGTGTT TTTTC-3'; bottom strand: 5'-TCGAGAAAAAACACGGATGATCTCCACATCATT CTCTCTCTTGAAGAGAATGATGTGGAGATCATCCGTGA-3') and scramble control shRNA (top strand: 5'-TTTTACTTGAGGCTCCCTCTGTAGCCTTCAAGA GAGGCTACAGAGGGAGCCTCAAGTAAATTTTTTC-3'; bottom strand: 5'-TCGA GAAAAAATTTACTTGAGGCTCCCTCTGTAGCCTCTCTTGAAGGCTACAGAGG GAGCCTCAAGTAAAA-3') were subcloned into pLL3.7 lentivirus vector with the restriction endonucleases $\mathrm{Hpal}$ and $\mathrm{Xhol}$ to generate pLL3.7-furin and pLL3.7control, respectively. pLL3.7-Furin or pLL3.7-control plasmids were cotransfected into 293 FT cells with packaging plasmids (VSVG, pRSV-Rev, pMDL g/p RRE) to produce the respective lentiviruses. Lentiviral particles were harvested at 2 days after transfection and concentrated by ultracentrifugation $(2 \mathrm{~h}$ at 39600 r.c.f., $4{ }^{\circ} \mathrm{C}$ ). After resuspension with the M16 medium, the concentration of the lentiviral vector was determined by infecting $293 \mathrm{~T}$ cells, followed by checking EGFP expression of infected cells by flow cytometry $48 \mathrm{~h}$ after infection.

Sexually mature CD1 female mice were superovulated using standard protocol and mated with the males. Embryos at blastocyst stage (E3.5) were collected and zona pellucidae were removed gently by $0.05 \%$ pronase (Sigma) incubation for 5 min. Each embryo was transferred to a $15 \mu \mathrm{l}$ M16 medium (Gibco) droplet containing $7.5 \times 10^{4}$ lentivirus. Infected blastocysts were examined under a laserscanning confocal microscope (Carl Zeiss LSM 710; Carl Zeiss Microlmaging $\mathrm{GmbH}$, Jena, Germany) to determine infection efficiency and specificity. After $8 \mathrm{~h}$ incubation, sibling blastocysts were implanted into the uteri of pseudopregnant female mice. Mice were killed at different stages of pregnancy. The whole placenta and fetus images were obtained by computer-assisted fluorescence anatomical lens (Zeiss; SteREO DiscoveryV.20). Placental tissues were frozen at $-80^{\circ} \mathrm{C}$ for protein extraction and cryosection. 
Western blotting. Whole-cell proteins were extracted with whole-cell lysis buffer (4 mM EGTA, $3 \mathrm{mM}$ EDTA, pH 8.0, $125 \mathrm{mM} \mathrm{NaF}, 0.5 \mathrm{mM} \mathrm{Na}_{3} \mathrm{VO}_{4}$ $2.5 \mu \mathrm{g} / \mathrm{ml}$ aprotinin, $25 \mu \mathrm{g} / \mathrm{ml}$ trypsin inhibitor, $25 \mu \mathrm{M}$ PMSF, $1 \%$ Triton X-100, $12.5 \mathrm{mM}$ HEPES, $1 \mathrm{mM}$ DTT). Proteins were quantified by the spectrophometry at $595 \mathrm{~nm}$ (Beckman DU530, Fullerton, CA, USA). In all, $50 \mu \mathrm{g}$ of extracted total proteins or proteins of spent media were subjected to SDS-PAGE gel electrophoresis and transferred electrophoretically onto a pure nitrocellulose blotting membrane (Pall Corporation, Pensacola, FL, USA). After being blocked with $5 \%$ skim milk, the membrane was sequentially incubated with primary antibodies against furin (ab3467; Abcam, Cambridge, UK), $\beta$-hCG (ab54410; Abcam), PC5 (ab39873; Abcam), PC7 (P9052; US Biological, Swampscott, MA, USA), IGF1R $\beta$ (sc-713; Santa Cruz Biotechnology), $\beta$-actin (ab8227; Abcam) or GAPDH (ab37187; Abcam), and horseradish peroxidase-conjugated secondary antibodies. Signals were developed using the Enhanced Chemiluminescence System (Pierce, Rockford, IL, USA). The intensity of the detected bands was quantified by laser densitometry and presented as a ratio of relative optical density of the band to the GAPDH $/ \beta$-actin band.

Transfection of siRNA or plasmids. Knockdown or overexpression of a gene was achieved by transfection of an siRNA or a plasmid using standard protocol. ${ }^{42,43}$

Immunofluorescence. Staining of furin, $\beta$-hCG, E-cadherin and CK7 was performed as described. ${ }^{42,43}$ Images were acquired using a Carl Zeiss LSM 710 confocal laser-scanning microscope with a $\times 63$ glycerol immersion objective, and image analysis was conducted using the Zeiss LSM Image Browser software. (Carl Zeiss Microlmaging GmbH).

Electron microscopy. Mouse placental tissues were collected and fixed with $4 \%$ glutaraldehyde in $0.1 \mathrm{~m}$ cacodylate buffer for $48 \mathrm{~h}$ at $4{ }^{\circ} \mathrm{C}$. Specimens were postfixed with $1 \%$ osmium tetroxide for $1 \mathrm{~h}$, dehydrated through a graded series of $50-100 \%$ ethanol, $100 \%$ propylene oxide, and then incubated in a $1: 1$ mixture of propylene oxide-epoxy resin, followed by embedding in resin. Ultrathin sections $(1 \mu \mathrm{m})$ were stained with uranyl acetate and lead citrate. Sections were examined with JEOL microscope (JEM01400; JEOL Co., Tokyo, Japan).

Statistical analysis. The bands from western blotting were quantified by MetaView Image Analyzing System (Version 4.50; Universal Imaging Corp., Downingtown, PA, USA). Each experiment was performed in triplicates. Results were presented as means \pm S.E.M. Statistical analysis was performed by one-way ANOVA and paired-sample $t$-test, which was performed by using the Statistical Package for Social Science (SPSS for Windows package release 10.0; SPSS Inc., Chicago, IL, USA). $P$-values of $<0.05$ were considered to be significant $\left({ }^{*} P<0.05 ;{ }^{* *} P<0.01\right)$.

\section{Conflict of Interest}

The authors declare no conflict of interest.

Acknowledgements. We thank Drs. Shengping Chen and Zhiying Gao for sample collection, and their patients for donating placental tissues. We also thank Jian-Hu Bai for technical assistance in generating placenta-specific knockdown mice. This study was supported by the National Key Basic Research Program of China (2011CB944400), the Strategic Priority Research Program of Chinese Academy of Sciences (XDA01020102) and grants from the Natural Science Foundation of China (81225004, 31271603 and 31171438).

1. Red-Horse K, Zhou Y, Genbacev O, Prakobphol A, Foulk R, McMaster M et al. Trophoblast differentiation during embryo implantation and formation of the maternal-fetal interface. $J$ Clin Invest 2004; 114: 744-754.

2. Huppertz B, Bartz C, Kokozidou M. Trophoblast fusion: fusogenic proteins, syncytins and ADAMs, and other prerequisites for syncytial fusion. Micron 2006; 37: 509-517.

3. Bhaumick B, George D, Bala RM. Potentiation of epidermal growth factor-induced differentiation of cultured human placental cells by insulin-like growth factor-I. J Clin Endocrinol Metab 1992; 74: 1005-1011.

4. Cohran V, Fang J, Milio L, Smith $\mathrm{CH}$, Fant M. Type I insulin-like growth factor receptors in the BeWo choriocarcinoma cell (b30 clone) during cell differentiation. Placenta 1996; 17 313-320.
5. Crocker IP, Strachan BK, Lash GE, Cooper S, Warren AY, Baker PN. Vascular endothelial growth factor but not placental growth factor promotes trophoblast syncytialization in vitro. J Soc Gynecol Invest 2001; 8: 341-346.

6. Leisser C, Saleh L, Haider S, Husslein H, Sonderegger S, Knofler M. Tumour necrosis factor-alpha impairs chorionic gonadotrophin beta-subunit expression and cell fusion of human villous cytotrophoblast. Mol Hum Reprod 2006; 12: 601-609.

7. Morrish DW, Bhardwaj D, Paras MT. Transforming growth factor beta 1 inhibits placental differentiation and human chorionic gonadotropin and human placental lactogen secretion. Endocrinology 1991; 129: 22-26.

8. Seidah NG, Chretien M. Eukaryotic protein processing: endoproteolysis of precursor proteins. Curr Opin Biotechnol 1997; 8: 602-607.

9. Seidah NG, Mayer G, Zaid A, Rousselet E, Nassoury N, Poirier S et al. The activation and physiological functions of the proprotein convertases. Int J Biochem Cell Biol 2008; 40: $1111-1125$

10. Seidah NG, Chretien M. Proprotein and prohormone convertases: a family of subtilases generating diverse bioactive polypeptides. Brain Res 1999; 848: 45-62.

11. Zhou Z, Shen T, Zhang BH, Lv XY, Lin HY, Zhu C et al. The proprotein convertase furin in human trophoblast: possible role in promoting trophoblast cell migration and invasion. Placenta 2009; 30: 929-938.

12. Duguay SJ, Lai-Zhang J, Steiner DF. Mutational analysis of the insulin-like growth factor I prohormone processing site. J Biol Chem 1995; 270: 17566-17574.

13. Lehmann M, Andre F, Bellan C, Remacle-Bonnet M, Garrouste F, Parat F et al. Deficien processing and activity of type I insulin-like growth factor receptor in the furin-deficient LoVo-C5 cells. Endocrinology 1998; 139: 3763-3771.

14. Siegfried G, Basak A, Cromlish JA, Benjannet S, Marcinkiewicz J, Chretien M et al. The secretory proprotein convertases furin, PC5, and PC7 activate VEGF-C to induce tumorigenesis. J Clin Invest 2003; 111: 1723-1732.

15. Chen CP, Chen LF, Yang SR, Chen CY, Ko CC, Chang GD et al. Functional characterization of the human placental fusogenic membrane protein syncytin 2. Biol Reprod 2008; 79: 815-823.

16. Cheynet V, Ruggieri A, Oriol G, Blond JL, Boson B, Vachot L et al. Synthesis, assembly, and processing of the Env ERVWE1/syncytin human endogenous retroviral envelope. $J$ Virol 2005; 79: 5585-5593.

17. Mi S, Lee X, Li X, Veldman GM, Finnerty $H$, Racie $L$ et al. Syncytin is a captive retroviral envelope protein involved in human placental morphogenesis. Nature 2000; 403: 785-789.

18. Roebroek AJ, Umans L, Pauli IG, Robertson EJ, van Leuven F, Van de Ven WJ et al. Failure of ventral closure and axial rotation in embryos lacking the proprotein convertase furin. Development 1998; 125: 4863-4876.

19. Thomas $G$. Furin at the cutting edge: from protein traffic to embryogenesis and disease. Nat Rev Mol Cell Biol 2002; 3: 753-766.

20. Scamuffa N, Siegfried G, Bontemps Y, Ma L, Basak A, Cherel G et al. Selective inhibition of proprotein convertases represses the metastatic potential of human colorectal tumor cells. J Clin Invest 2008; 118: 352-363.

21. Forbes K, Westwood M. The IGF axis and placental function. a mini review. Horm Res 2008; 69: 129-137.

22. Baczyk D, Drewlo S, Proctor L, Dunk C, Lye S, Kingdom J. Glial cell missing-1 transcription factor is required for the differentiation of the human trophoblast. Cell Death Differ 2009; 16: 719-727.

23. Okada $Y$, Ueshin $Y$, Isotani A, Saito-Fujita $T$, Nakashima $H$, Kimura $K$ et al. Complementation of placental defects and embryonic lethality by trophoblast-specific lentiviral gene transfer. Nat Biotechnol 2007; 25: 233-237.

24. Rubinstein E, Ziyyat A, Wolf JP, Le Naour F, Boucheix $C$. The molecular players of spermegg fusion in mammals. Semin Cell Dev Biol 2006; 17: 254-263.

25. Vignery A. Osteoclasts and giant cells: macrophage-macrophage fusion mechanism. Int $J$ Exp Pathol 2000; 81: 291-304

26. Roberts L, Sebire NJ, Fowler D, Nicolaides KH. Histomorphological features of chorionic villi at 10-14 weeks of gestation in trisomic and chromosomally normal pregnancies. Placenta 2000; 21: 678-683.

27. Malassine A, Frendo JL, Evain-Brion D. Trisomy 21-affected placentas highlight prerequisite factors for human trophoblast fusion and differentiation. Int J Dev Biol 2010; 54: $475-482$.

28. Gauster M, Siwetz M, Orendi K, Moser G, Desoye G, Huppertz B. Caspases rather than calpains mediate remodelling of the fodrin skeleton during human placental trophoblast fusion. Cell Death Differ 2010; 17: 336-345.

29. Heazell AE, Moll SJ, Jones CJ, Baker PN, Crocker IP. Formation of syncytial knots is increased by hyperoxia, hypoxia and reactive oxygen species. Placenta 2007; 28(Suppl A): S33-S40.

30. Huppertz B. Placental origins of preeclampsia: challenging the current hypothesis. Hypertension 2008; 51: 970-975.

31. Yuasa K, Masuda T, Yoshikawa C, Nagahama M, Matsuda Y, Tsuji A. Subtilisin-like proprotein convertase PACE4 is required for skeletal muscle differentiation. $J$ Biochem 2009; 146: 407-415.

32. Rossant J, Cross JC. Placental development: lessons from mouse mutants. Nat Rev Genet 2001; 2: 538-548.

33. Hemberger M, Cross JC. Genes governing placental development. Trends Endocrinol Metab 2001; 12: 162-168. 
34. Cross JC, Nakano H, Natale DR, Simmons DG, Watson ED. Branching morphogenesis during development of placental villi. Differentiation 2006; 74: 393-401.

35. Malashicheva A, Kanzler B, Tolkunova E, Trono D, Tomilin A. Lentivirus as a tool for lineage-specific gene manipulations. Genesis 2007; 45: 456-459.

36. Kumasawa K, Ikawa M, Kidoya H, Hasuwa H, Saito-Fujita T, Morioka Y et al. Pravastatin induces placental growth factor (PGF) and ameliorates preeclampsia in a mouse model. Proc Natl Acad Sci USA 2011; 108: 1451-1455

37. Guzman-Ayala M, Ben-Haim N, Beck S, Constam DB. Nodal protein processing and fibroblast growth factor 4 synergize to maintain a trophoblast stem cell microenvironment. Proc Natl Acad Sci USA 2004; 101: 15656-15660.

38. Hughes M, Dobric N, Scott IC, Su L, Starovic M, St-Pierre B et al. The Hand1, Stra13 and $\mathrm{Gcm} 1$ transcription factors override FGF signaling to promote terminal differentiation of trophoblast stem cells. Dev Biol 2004; 271: 26-37.

39. Li H, Dakour J, Kaufman S, Guilbert LJ, Winkler-Lowen B, Morrish DW. Adrenomedullin is decreased in preeclampsia because of failed response to epidermal growth factor and impaired syncytialization. Hypertension 2003; 42: 895-900.

40. Wang $\mathrm{H}$, Li Q, Shao L, Zhu C. Expression of matrix metalloproteinase-2, $-9,-14$, and tissue inhibitors of metalloproteinase- $1,-2,-3$ in the endometrium and placenta of rhesus monkey (Macaca mulatta) during early pregnancy. Biol Reprod 2001; 65: 31-40.
41. Rubinson DA, Dillon CP, Kwiatkowski AV, Sievers C, Yang L, Kopinja J et al. A lentivirusbased system to functionally silence genes in primary mammalian cells, stem cells and transgenic mice by RNA interference. Nat Genet 2003; 33: 401-406.

42. Fu J, Lv X, Lin H, Wu L, Wang R, Zhou Z et al. Ubiquitin ligase cullin 7 induces epithelialmesenchymal transition in human choriocarcinoma cells. J Biol Chem 2010; 285 10870-10879.

43. Kawaguchi N, Sundberg C, Kveiborg M, Moghadaszadeh B, Asmar M, Dietrich N et al. ADAM12 induces actin cytoskeleton and extracellular matrix reorganization during early adipocyte differentiation by regulating beta1 integrin function. J Cell Sci2003; 116(Part 19): 3893-3904.

\footnotetext{
(c) (i) $(\theta)($ Cell Death and Disease is an open-access journal published by Nature Publishing Group. This work is licensed under a Creative Commons Attribution-NonCommercialNoDerivs 3.0 Unported License. To view a copy of this license, visit http://creativecommons.org/licenses/by-nc-nd/3.0/
}

Supplementary Information accompanies this paper on Cell Death and Disease website (http://www.nature.com/cddis) 